\title{
Bases genéticas de milho e alterações no plano experimental
}

\author{
Thomas Newton Martin ${ }^{(1)}$, Lindolfo Storck ${ }^{(1)}$, Alessandro Dal'Col Lúcio(1), Melissa Pisaroglo de Carvalho(1) \\ e Paula Machado dos Santos ${ }^{(1)}$
}

\begin{abstract}
(1)Universidade Federal de Santa Maria, Dep. de Fitotecnia, Av. Roraima s/no, Bairro Camobi, CEP 97105-900 Santa Maria, RS. E-mail: thomas.martin@bol.com.br, storck@ccr.ufsm.br, adlucio@ccr.ufsm.br, pisaroglo@bol.com.br, paula.san@pop.com.br
\end{abstract}

\begin{abstract}
Resumo - Resultados experimentais seguros exigem um plano experimental apropriado, obtido através da utilização de adequado tamanho e forma de parcelas, número de repetições e de amostras. O objetivo deste trabalho foi avaliar a influência de diferentes bases genéticas de milho, híbrido simples (DAS 9560), duplo (AG 6018), triplo (AG 303) e variedade cultivada (Pampa), sobre a estimativa do tamanho de parcela experimental e do número de repetições. $\mathrm{O}$ delineamento experimental foi de blocos ao acaso com quatro repetições e amostragem nas parcelas. As parcelas experimentais foram constituídas por oito fileiras com 12 metros de comprimento, formando 96 unidades básicas. O tamanho de parcela e o número de repetições foram estimados por meio dos métodos de Lessman \& Atkins e de Hatheway. O tamanho ótimo de parcela variou entre 4,5 $\mathrm{m}^{2}$ (híbrido simples) e 7,2 $\mathrm{m}^{2}$ (híbrido duplo). A heterogeneidade do solo e a variabilidade genética influenciam no tamanho ótimo de parcela e número de repetições necessárias.
\end{abstract}

Termos para indexação: Zea mays, índice de heterogeneidade do solo, tamanho de parcela, número de repetições.

\section{Maize genetic variability and modifications on the experimental plan}

\begin{abstract}
An adequate experimental plan is necessary to get high quality data. Experimental plan ensures correct plot size and shape as well as adequate number of replications and samples. The objective of this work was to verify if maize genetic variability could interfere in the size of experimental plots and number of replications. Single (DAS 9560), double (AG 6018) and triple (AG 303) crosses and the open pollinated variety (Pampa) were evaluated. The experiment was carried out in a randomized complete block design with four replications. Plot samples comprised experimental plots with eight rows of $12 \mathrm{~m}$ with 96 basic units. Lessman \& Atkins and Hatheway methods were used to estimate optimum plot size and replication number. The best plot size varied from $4.5 \mathrm{~m}^{2}$ (simple hybrid) to $7.2 \mathrm{~m}^{2}$ (double hybrid). Soil heterogeneity and genetic variability change plot size and number of replications requested.
\end{abstract}

Index terms: Zea mays, soil heterogeneity, plot size, number of replications.

\section{Introdução}

Os programas de melhoramento das empresas produtoras de sementes de milho são muito dinâmicos, disponibilizando, anualmente, grande diversidade de genótipos para os agricultores. A base genética dos milhos mais cultivados são os híbridos de linhagem (simples, duplo e triplo) e as variedades cultivadas. Cada base genética é adaptada a condições específicas de ambiente, devido à variabilidade presente na sua constituição. A variabilidade genética das plantas de milho nessas cultivares aumenta do híbrido simples para o híbrido triplo, híbrido duplo e, finalmente, a variedade cultivada (Naspolini Filho, 1975; Lemos, 1976).

Le Clerg et al. (1962) verificaram que, entre os cereais, a cultura do milho possuía baixa precisão experi- mental, portando-se de maneira superior apenas quando comparada com plantas arbóreas. Essa baixa precisão experimental foi confirmada 35 anos depois por Lúcio (1997), em ensaios de competição de cultivares (milho, arroz, soja e trigo) no Rio Grande do Sul.

A precisão experimental é afetada pela heterogeneidade do solo e do material experimental, competições intraparcelar e interparcelar, amostragem na parcela, atendimento às pressuposições do modelo matemático, entre outros fatores (Storck et al., 2000). Parcelas situadas lado a lado possuem diferenças em virtude da heterogeneidade do solo (Loma, 1955; Le Clerg et al., 1962; Gomez \& Gomez, 1984). Além disso, a heterogeneidade do material experimental, em conjunto com a heterogeneidade do solo, influencia diretamente o número de repetições necessário e o tama- 
nho adequado da parcela experimental (Steel et al., 1997). Caso o material experimental não seja homogêneo, uma certa quantidade de material, numa unidade experimental, não representa por amostragem a mesma quantidade em outra unidade, o que irá aumentar o erro experimental (Storck et al., 2000).

Em relação à cultura do milho, a estimativa do tamanho ótimo de parcela usando um ensaio em branco foi realizada por Storck (1979). O autor trabalhou com híbrido duplo e determinou, por meio de métodos de estimação, que o melhor tamanho de parcela constituía-se de uma fileira simples com 5,0 m, ou duas fileiras de 2,5 m no sentido contrário às filas do ensaio. Resende \& Souza Júnior (1997), no entanto, determinaram que, para avaliação de progênies, são necessárias três repetições com 20 plantas para solo do cerrado e 15 plantas para solo fértil, estudando 200 progênies de meios-irmãos da variedade cultivada BR 108.

O objetivo deste trabalho foi verificar se diferentes bases genéticas de milho alteram a estimativa do tamanho ótimo de parcela e do número de repetições.

\section{Material e Métodos}

O experimento foi conduzido durante o ano agrícola 2001/2002, na UFSM. O local possui relevo plano ondulado e solo classificado como Brunizem Hidromórfico, pertencente à Unidade de Mapeamento Santa Maria (Embrapa, 1999). A adubação no experimento foi realizada segundo as Recomendações de Adubação e Calagem para os Estados do Rio Grande do Sul e Santa Catarina (Comissão de Fertilidade do Solo - RS/SC, 1995).

O delineamento experimental foi o de blocos ao acaso com quatro repetições. Os tratamentos foram constituídos por quatro genótipos de milho, DAS 9560 (híbrido simples), AG 6018 (híbrido triplo), AG 303 (híbrido duplo) e Pampa (variedade cultivada). A unidade experimental (parcela) foi composta por oito fileiras de $12 \mathrm{~m}$ de comprimento, distanciados $0,8 \mathrm{~m}$ entre si. Nas avaliações, as parcelas ou unidades experimentais foram divididas em 96 subunidades (unidades básicas) de um metro linear, circundadas por $4 \mathrm{~m}$ de bordadura lateral.

A semeadura do milho foi realizada no dia 9 de novembro de 2001, colocando-se, manualmente, duas sementes por cova, a uma profundidade de $5 \mathrm{~cm}$. Foram semeadas cinco covas eqüidistantes por metro, que, após o desbaste, resultaram na densidade de 62.500 plantas por hectare. O controle de plantas daninhas foi realizado com atrazine + simazine, nas dosagens de 1,625 $\mathrm{g} \mathrm{ha}^{-1}$ de i.a. e 1,625 $\mathrm{g} \mathrm{ha}^{-1}$ de i.a., respectivamente. $\mathrm{O}$ controle de insetos foi efetuado com deltamethrine a $5 \mathrm{~g} \mathrm{ha}^{-1}$ de i.a. As colheitas foram realizadas entre 10 e 16/5/2002 com o teor de água dos grãos em aproximadamente $20 \%$.

Em cada subunidade básica (1 m), foi avaliado o rendimento de grãos a $13 \%$ de umidade (RG), o número de plantas (NP) e o número de espigas (NE). A análise estatística baseou-se no delineamento de blocos ao acaso com amostragem nas parcelas. As pressuposições do modelo matemático quanto à variável rendimento de grãos - aleatoriedade dos erros estimados, homogeneidade da variância dos erros entre os genótipos, aditividade do modelo matemático e normalidade da distribuição dos erros - foram verificadas conforme aplicações de Marques (1999).

Para cada parcela principal, foram planejados tipos de parcela de diferentes tamanhos. Cada tipo de parcela apresentou X1 unidades básicas de comprimento (colunas) e X2 unidades básicas de largura (filas), formadas pelo agrupamento de unidades contíguas, de modo que X1×X2 correspondem a X (tamanho da parcela em número de unidades básicas). Dessa maneira, o número de repetições de cada tipo de parcela ficou limitado pela área total da parcela principal, sendo planejados os tipos de parcela $(\mathrm{X} 1 \times \mathrm{X} 2)$ : $1 \times 1,2 \times 1,3 \times 1,4 \times 1,6 \times 1,2 \times 2$, $3 \times 2,4 \times 2,6 \times 2,4 \times 3,4 \times 4$ e $6 \times 4$. Estabeleceram-se os seguintes parâmetros: $\mathrm{N}$, número de parcelas, com $\mathrm{X}$ unidades básicas (UB) de tamanho calculado por N, 96/X; $\mathrm{M}(\mathrm{x})$, média das parcelas com X UB de tamanho; $\mathrm{V}(\mathrm{x})$, variância entre parcelas de X UB de tamanho; VU(x), variância por UB, calculada entre as parcelas de X UB, $\mathrm{V}(\mathrm{x}) / \mathrm{X}^{2} \mathrm{e} C \mathrm{~V}(\mathrm{x})$, coeficiente de variação entre as parcelas de X UB de tamanho. Tendo por base esses parâmetros, foi estimado o índice de heterogeneidade do solo (b), segundo a relação empírica $\mathrm{VU}(\mathrm{x})=\mathrm{V} 1 / \mathrm{X}^{\mathrm{b}}$, de Smith (1938), para o valor do rendimento de grãos, em que $\mathrm{VU}(\mathrm{x})$ é a variância do valor observado por $\mathrm{UB}$, calculada entre as parcelas com X UB; $\mathrm{V}_{1}$ é a variância dos valores de parcelas constituídas de uma UB; $X$ é o número de UB que compõe a parcela no iésimo tipo de parcela considerado, $\mathrm{i}=1,2,3, \ldots, 12$; e b é o índice de heterogeneidade do solo.

$\mathrm{O}$ valor de $\mathrm{b}$ é estimado por um coeficiente de regressão linear, através da logaritmização da função $\mathrm{VU}(\mathrm{x})=\mathrm{V} 1 / \mathrm{X}^{\mathrm{b}}$, ou seja: $\log \mathrm{VU}(\mathrm{x})=\log \mathrm{V} 1-\mathrm{b} \log \mathrm{X}$, 
cuja estimação foi ponderada pelos graus de liberdade, associados aos 12 tipos de parcelas (Steel et al., 1997).

Às estimativas dos índices de heterogeneidade do solo (b) foi aplicado o teste de paralelismo (Seber, 1976) entre as repetições dos genótipos e entre os genótipos, a fim de testar a hipótese de paralelismo das regressões $\left(\mathrm{H}_{0}: \mathrm{b}_{1}=\mathrm{b}_{2}=\ldots=\mathrm{b}_{\mathrm{k}}\right)$. A estatística do teste é:

$$
\mathrm{F}_{\text {cal }}=\frac{\left(\mathrm{SQE}_{\mathrm{H} 0}-\sum_{\mathrm{k}=1}^{\mathrm{K}} \mathrm{SQE}\right) /(\mathrm{K}-1)}{\sum_{\mathrm{k}=1}^{\mathrm{K}} \mathrm{SQE} /(\mathrm{n}-2 \mathrm{~K})},
$$

em que $\mathrm{SQE}_{\mathrm{H} 0}$ é a soma de quadrados do erro conjunto; $\sum_{\mathrm{k}=1}^{\mathrm{K}} \mathrm{SQE}$ é o somatório da soma de quadrados do erro de cada regressão; $\mathrm{K}$ é o número de regressões comparadas; e n é o número total de unidades básicas. Quando $F_{\text {cal }}$ é maior que o valor de $F_{\text {tab }}\left(\alpha\right.$; GL $\mathrm{SQE}_{\mathrm{SQ}}$; $\left.\mathrm{GL}_{\mathrm{SQE}_{\mathrm{HO}}}\right)$, não existe paralelismo entre os coeficientes de heterogeneidade do solo.

O tamanho da parcela foi estimado por meio do método da máxima curvatura modificado, proposto por Lessman \& Atkins (1963). O ponto de máxima curvatura de $\mathrm{CV}=\mathrm{A} / \mathrm{X}^{\mathrm{B}}$ define o tamanho ótimo da parcela, que é dado pela função: $\log \mathrm{X}_{\text {ótimo }}=\left(\frac{1}{2 \mathrm{~B}+2}\right) \log \left(\frac{\mathrm{A}^{2} \mathrm{~B}^{2}(2 \mathrm{~B}+1)}{(\mathrm{B}+2)}\right)$.

O número de repetições foi determinado através do método de Hatheway (1961), cuja fórmula para estimálo em um experimento é $r=\frac{2(\mathrm{t} 1+\mathrm{t} 2)^{2} \mathrm{~A}^{2}}{\mathrm{~d}^{2} \mathrm{X}^{\mathrm{b}}}$,

em que $\mathrm{r}$ é o número de repetições requerido para detectar diferenças de d unidades; $d$ é a diferença verdadeira entre duas médias de tratamento, expressa em porcentagem da média; A é a estimativa do coeficiente de variação em porcentagem para uma unidade básica; t1 é o valor da Tabela de t para testes de significância (bilateral a 5\%); e t2 é o valor da Tabela de t correspondente a 2 (1-P), em que P é a probabilidade de se obter resultados significativos $(0,8)$; $\mathrm{X}$ é o tamanho ótimo da parcela; e b é o índice de heterogeneidade do solo.

\section{Resultados e Discussão}

A emergência do milho ocorreu seis dias após a semeadura, de maneira uniforme, quanto aos genótipos DAS 9560, AG 6018 e AG 303. Em relação à varieda- de cultivada Pampa, não houve germinação uniforme nem suficiente, justificando a sua ressemeadura. $\mathrm{O}$ crescimento e o desenvolvimento das plantas foram satisfatórios em virtude do eficiente controle de plantas daninhas e de pragas.

Quanto a variável rendimento de grãos, ajustada a $13 \%$ de umidade, todas as pressuposições do modelo matemático foram atendidas. Dessa forma, pode-se dizer que a qualidade dos experimentos foi garantida para corretos testes de hipóteses (Storck et al., 2000). Não houve efeito significativo de blocos sobre as variáveis estudadas, além de existir pelo menos um contraste significativo entre genótipos em todas as variáveis avaliadas (Tabela 1). Mesmo os blocos sendo homogêneos em relação às variáveis rendimento de grãos, número de plantas e de espigas, verificou-se que houve interação bloco x genótipo (erro experimental) em todas as variáveis, indicando que a variação existente entre parcelas com o mesmo tratamento não é apenas proveniente da variação amostral mas, também, da resposta diferencial das cultivares em cada bloco. Assim, a baixa precisão experimental verificada (alto $\mathrm{CVe}$ ) na variável rendimento de grãos pode ser atribuída à significância do erro experimental, proveniente sobretudo da interação bloco $\mathrm{x}$ genótipo.

Os índices de heterogeneidade do solo, quanto à variável rendimento de grãos (Tabela 2), apresentaram uma grande variação entre as 16 parcelas estudadas, variando de 0,147 a 2,175, sendo o índice médio (conjunto das 16 parcelas) igual a 0,941 . Isso significa grande heterogeneidade do solo, ou baixa correlação entre as subunidades (parcelas) adjacentes, indicando que as parcelas experimentais, neste local, devem ser maiores, a fim de evitar que um tratamento incida sobre um local diferente de outro.

Em testes com os índices de heterogeneidade do solo entre as repetições do mesmo genótipo, verificou-se que não apresentam comportamento paralelo em todos os genótipos testados. O teste de paralelismo sobre os índices de heterogeneidade médios (solo e genótipo) resultou na ausência de paralelismo de resposta, indicando assim a necessidade do uso de um tamanho de parcela para cada localidade avaliada e também para cada genótipo.

O tamanho da parcela variou de 1,44 a 8,98 unidades básicas, ou seja, de 7 a 45 plantas, sendo que a média para os genótipos, em UB, foi 4,99 (DAS 9560), 4,46 (AG 6018), 6,50 (AG 303) e 6,48 (Pampa), o que 
Tabela 1. Análise da variância segundo o delineamento de blocos ao acaso com amostragem na parcela, graus de liberdade (GL), quadrados médios e médias por genótipo das variáveis rendimento de grãos $(\mathrm{RG})$, número de plantas ha ${ }^{-1}(\mathrm{NP})$, número de espigas ha-1 $(\mathrm{NE})$, média e coeficiente de variação do erro experimental (CVe) e coeficiente de variação do erro amostral (CVea) ${ }^{(1)}$.

\begin{tabular}{lcccc}
\hline Fonte de variação & GL & RG $\left(\mathrm{t} \mathrm{ha}^{-1}\right)$ & NP (milhares) & NE (milhares) \\
\hline Blocos & 3 & $90,64^{\mathrm{ns}}$ & $511,71^{\mathrm{ns}}$ & $336,77^{\mathrm{ns}}$ \\
Genótipos & 3 & $1.312,15^{*}$ & $17.261,92^{*}$ & $37.058,51^{*}$ \\
Erro experimental & 9 & $48,25^{*}$ & $243,69^{*}$ & $341,20^{*}$ \\
Erro amostral & 1.520 & 4,18 & 120,53 & 153,53 \\
\hline DAS 9560 & - & $8,52 \mathrm{a}$ & $55,46 \mathrm{a}$ & $53,71 \mathrm{a}$ \\
AG 6018 & - & $7,22 \mathrm{ab}$ & $53,05 \mathrm{a}$ & $50,98 \mathrm{ab}$ \\
AG 303 & - & $6,18 \mathrm{~b}$ & $53,58 \mathrm{a}$ & $47,95 \mathrm{~b}$ \\
Pampa & - & $4,13 \mathrm{c}$ & $40,78 \mathrm{~b}$ & $31,80 \mathrm{c}$ \\
\hline Média & - & 6,51 & 50,72 & 46,11 \\
Cve & - & 106,57 & 30,77 & 40,06 \\
CVea & - & 31,39 & 21,64 & 26,87 \\
\hline
\end{tabular}

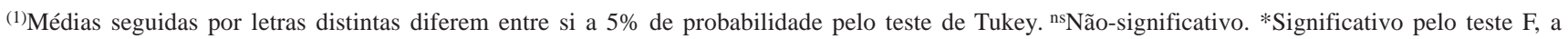
$5 \%$ de probabilidade.

Tabela 2. Estimativa dos parâmetros da relação $\mathrm{CV}(\mathrm{x})=\mathrm{A} / \mathrm{X}^{\mathrm{B}}$, coeficiente de determinação $\left(\mathrm{r}^{2}\right)$, índice de heterogeneidade do solo ( $(\hat{b})$, estimativa do tamanho ótimo de parcela através do método de máxima curvatura modificado (TOP), média $(\overline{\mathrm{X}})$ e valores máximos dos parâmetros $\left(\mathrm{M}_{\mathrm{x}}\right)$, por genótipo e no global $(\mathrm{MG})$ e máximo global $\left(\mathrm{M}_{\mathrm{x}} \mathrm{G}\right)$, com os respectivos números de repetições para a diferença verdadeira entre duas médias de tratamentos, expressa em porcentagem da média (d\%).

\begin{tabular}{|c|c|c|c|c|c|c|c|c|c|c|}
\hline \multirow[t]{2}{*}{ Variável } & \multirow[t]{2}{*}{ Bloco } & \multirow[t]{2}{*}{ A } & \multirow[t]{2}{*}{ B } & \multirow[t]{2}{*}{$\mathrm{r}^{2}$} & \multirow[t]{2}{*}{$\hat{b}$} & \multirow[t]{2}{*}{ TOP } & \multicolumn{4}{|c|}{$\mathrm{d} \%$} \\
\hline & & & & & & & 5 & 10 & 20 & 40 \\
\hline & \multicolumn{10}{|c|}{ DAS 9560 - Híbrido Simples } \\
\hline & 1 & $24,68 * *$ & $0,57 * *$ & 0,89 & 1,141 & 5,08 & - & - & - & - \\
\hline & 2 & $28,99 * *$ & $0,56 * *$ & 0,88 & 1,121 & 5,62 & - & - & - & - \\
\hline & 3 & $21,81 * *$ & $0,53 * *$ & 0,80 & 1,069 & 4,64 & - & - & - & - \\
\hline & 4 & $22,70 * *$ & $0,47 * *$ & 0,95 & $0,939 *$ & 4,61 & - & - & - & - \\
\hline $\bar{X}$ & - & 24,55 & - & - & $1,067 * *$ & 4,99 & 69 & 18 & 5 & 2 \\
\hline \multirow{6}{*}{$\mathrm{M}_{\mathrm{x}}$} & - & 28,99 & - & - & - & 5,62 & 75 & 19 & 5 & 2 \\
\hline & \multicolumn{10}{|c|}{ AG 6018 - Híbrido Triplo } \\
\hline & 1 & $26,96 * *$ & $0,51 * *$ & 0,90 & 1,024 & 5,28 & - & - & - & - \\
\hline & 2 & $19,51 * *$ & $0,48 * *$ & 0,74 & 0,959 & 4,19 & - & - & - & - \\
\hline & 3 & $27,14^{*}$ & $0,07 *$ & 0,50 & 0,147 & 1,44 & - & - & - & - \\
\hline & 4 & $42,84 * *$ & $0,41 * *$ & 0,95 & $0,828^{*}$ & 6,92 & - & - & - & - \\
\hline \multirow{6}{*}{$\begin{array}{l}\bar{X} \\
M_{x}\end{array}$} & - & 29,11 & - & - & 0,739 & 4,46 & 179 & 45 & 12 & 3 \\
\hline & - & 42,84 & - & - & - & 6,92 & 162 & 41 & 11 & 3 \\
\hline & \multicolumn{10}{|c|}{ AG 303 - Híbrido Duplo } \\
\hline & 1 & $37,70 * *$ & $0,38 * *$ & 0,75 & 0,761 & 6,17 & - & - & - & - \\
\hline & 2 & $28,88 *$ & $0,24 *$ & 0,65 & 0,478 & 4,02 & - & - & - & - \\
\hline & 3 & $88,67 * *$ & $1,09 * *$ & 0,78 & 2,175 & 8,98 & - & - & - & - \\
\hline \multirow{8}{*}{$\begin{array}{l}\bar{X} \\
M_{x}\end{array}$} & 4 & $39,40 * *$ & $0,67 * *$ & 0,84 & $1,348^{*}$ & 6,82 & - & - & - & - \\
\hline & - & 48,66 & - & - & 1,190 & 6,50 & 163 & 41 & 11 & 3 \\
\hline & - & 88,67 & - & - & - & 8,98 & 43 & 11 & 3 & 1 \\
\hline & \multicolumn{10}{|c|}{ Pampa - Variedade cultivada } \\
\hline & 1 & $40,81 * *$ & $0,28 * *$ & 0,89 & 0,562 & 5,80 & - & - & - & - \\
\hline & 2 & $40,94 * *$ & $0,24 * *$ & 0,88 & 0,480 & 5,34 & - & - & - & - \\
\hline & 3 & $50,01 * *$ & $0,61 * *$ & 0,94 & 1,219 & 7,95 & - & - & - & - \\
\hline & 4 & $45,68^{*}$ & $0,34 *$ & 0,94 & $0,792 *$ & 6,84 & - & - & - & - \\
\hline \multirow{2}{*}{$\begin{array}{l}\bar{X} \\
M_{x}\end{array}$} & - & 44,36 & - & - & 0,764 & 6,48 & 301 & 76 & 19 & 5 \\
\hline & - & 50,51 & - & - & - & 7,95 & 130 & 33 & 9 & 3 \\
\hline MG & - & - & - & - & - & - & 170 & 43 & 11 & 3 \\
\hline $\mathrm{M}_{\mathrm{x}} \mathrm{G}$ & - & 88,67 & 1,09 & - & 2,175 & 8,98 & 43 & 11 & 3 & 1 \\
\hline
\end{tabular}

$*$ e $* *$ Significativamente diferente de zero a $5 \%$ e a $1 \%$ de probabilidade, respectivamente. 
equivale, respectivamente, a 25, 22, 33 e 33 plantas por parcela (Tabela 2). A utilização do maior tamanho de parcela encontrado para cada genótipo é indicada a fim de garantir melhor proteção à qualidade de informações. Os maiores valores de tamanho de parcela encontrados para os genótipos foram $5,62\left(4,50 \mathrm{~m}^{2}\right), 6,92\left(5,54 \mathrm{~m}^{2}\right), 8,98\left(7,18 \mathrm{~m}^{2}\right) \mathrm{e}$ $7,95\left(6,36 \mathrm{~m}^{2}\right)$ UB para os representantes dos genótipos híbrido simples, híbrido triplo, híbrido duplo e variedade cultivada, respectivamente, correspondendo a 29, 35, 45 e 40 plantas por parcela.

Com base nos resultados, podem-se agrupar os experimentos de competição de cultivares conforme a base genética em grupos distintos - ou com afinidades do mesmo tamanho de parcela e número de repetições -, também sugerido por Arnold \& Jenkins (1932). Como segunda opção, a adoção do maior tamanho ótimo de parcela encontrado em todas as repetições dos quatro genótipos utilizados. A utilização de 45 plantas por parcela permite uma padronização do tamanho de parcela, entretanto o experimento necessitaria de maior área experimental, mais recursos financeiros e mão-de-obra.

Com base nos resultados do número de repetições (Tabela 2), conforme as médias estimadas, pode-se verificar que para qualquer nível de precisão, o híbrido simples (DAS 9560) possui, em média, menor exigência no número de repetições. Os genótipos AG 6018 e AG 303, ao contrário, são mais exigentes em número de repetições que o híbrido simples, sendo que o genótipo AG 6018, em todos os níveis de precisão, exigiu um número de repetições levemente superior que o híbrido AG 303. A variedade cultivada (Pampa) apresentou os maiores números de repetições para um mesmo nível de precisão, o que a caracteriza como um material de grande variabilidade. Com o uso dos valores máximos obtidos em cada repetição, para cada genótipo, obtém-se uma melhor proteção estatística para os resultados. Assim, numa variação de d de 5 a 40, o número de repetições oscilou de 43 até 1 parcela, respectivamente. Esses valores extremos não permitem uma execução correta dos resultados dos experimentos. No segundo caso (1 parcela), não há repetições para estimativas da média e demais parâmetros e, no primeiro caso, a demanda de área e investimentos são demasiados. Nos intervalos de confiança de $10 \%$ e $20 \%$, obtiveram-se resultados mais coerentes, possibilitando a im- plantação dos experimentos com 11 e 3 repetições, respectivamente.

Dos seis pares de genótipos, quatro apresentam índice de heterogeneidade do solo não-paralelos (Tabela 3). Dos genótipos comparados pelo teste de paralelismo, verifica-se que os índices de heterogeneidade do solo não possuem um comportamento paralelo nas seguintes combinações: DAS 9560 (HS) e AG 6018 (HT); DAS 9560 (HS) e Pampa (VAR); AG 6018 (HT) e AG 303 (HD); e AG 303 (HD) e Pampa (VAR). A ausência de parelelismo nestas combinações pode decorrer de fatores genéticos - uma vez que quatro bases genéticas distintas são avaliadas - e, também, das condições ambientais do solo, que alteram o índice de heterogeneidade. No entanto, foi possível verificar duas combinações de genótipos com desempenho semelhante, sendo elas: DAS 9560 (HS) e AG 303 (HD); e AG 6018 (HT) e Pampa (VAR). Devido à provável semelhança na constituição dos materiais experimentais e à proximidade entre os índices de heterogeneidade do solo, o resultado obtido foi de paralelismo entre estes índices.

Tabela 3. Teste de paralelismo dos índices de heterogeneidade do solo ( $\hat{b})$, entre pares de genótipos, graus de liberdade do resíduo (GLr), somatório de quadrados do resíduos ( $\mathrm{SQr})$ e valor $\mathrm{F}$ calculado $\left(\mathrm{F}_{\mathrm{cal}}\right)$.

\begin{tabular}{lcrcc}
\hline Classe & GLr & SQr & $\hat{b}$ & $\mathrm{~F}_{\text {cal }}$ \\
\hline DAS 9560 (HS) & 46 & 0,61776759 & 1,068 & - \\
AG 6018 (HT) & 46 & 5,19234811 & 0,736 & - \\
Soma & 92 & 5,81011570 & - & - \\
Global & 94 & 7,16152848 & 0,903 & $21,399^{*}$ \\
\hline DAS 9560 (HS) & 46 & 0,61776759 & 1,068 & - \\
AG 303 (HD) & 46 & 5,53169948 & 1,190 & - \\
Soma & 92 & 6,14946707 & - & - \\
Global & 94 & 6,31602701 & 1,129 & $2,492^{\text {ns }}$ \\
\hline DAS 9560 (HS) & 46 & 0,61776759 & 1,068 & - \\
Pampa (VAR) & 46 & 1,58503377 & 0,765 & - \\
Soma & 92 & 2,20280136 & - & - \\
Global & 94 & 7,16152848 & 0,903 & $207,101^{*}$ \\
\hline AG 6018 (HT) & 46 & 5,19234811 & 0,736 & - \\
AG 303 (HD) & 46 & 5,53169948 & 1,190 & - \\
Soma & 92 & 10,72404759 & - & - \\
Global & 94 & 11,47219443 & 0,965 & $6,418^{*}$ \\
\hline AG 6018 (HT) & 46 & 5,19234811 & 0,736 & - \\
Pampa (VAR) & 46 & 1,58503377 & 0,765 & - \\
Soma & 92 & 6,77738188 & - & - \\
Global & 94 & 6,90216825 & 0,752 & $1,694^{\text {ns }}$ \\
\hline AG 303 (HD) & 46 & 5,53169948 & 1,190 & - \\
Pampa (VAR) & 46 & 1,58503377 & 0,765 & - \\
Soma & 92 & 7,11673325 & - & - \\
Global & 94 & 7,45483438 & 0,978 & $4,370^{*}$ \\
\hline
\end{tabular}

${ }^{n}$ Não-significativo. *Significativo pelo teste $\mathrm{F}$ a $5 \%$ de probabilidade. 


\section{Conclusão}

A heterogeneidade do solo e a variabilidade genética são responsáveis pela diversidade do tamanho ótimo de parcela e do número de repetições, num mesmo nível de precisão.

\section{Agradecimentos}

Ao CNPq e à Fapergs, pelo auxílio financeiro.

\section{Referências}

ARNOLD, L.E.; JENKINS, M.T. The relative variability of corn crosses and varieties. Journal of the American Society of Agronomy, v. 24, p.868-871, 1932.

COMISSÃO DE FERTILIDADE DO SOLO-RS/SC. Recomendações de adubação e calagem para o Estado do Rio Grande do Sul e Santa Catarina. 3.ed. Passo Fundo: SBCC-Núcleo Regional Sul, Embrapa-CNPT, 1995. 223p.

EMBRAPA. Centro Nacional de Pesquisa de Solos (Rio de Janeiro, RJ). Sistema brasileiro de classificação dos solos. Brasília: Embrapa-SPI; Embrapa-CNPS, 1999. 412p.

GOMEZ, K.A.; GOMEZ, A.A. Statistical procedures for agricultural research. 2nd ed. New York: John Wiley, 1984. 680p.

HATHEWAY, W.H. Convenient plot size. Agronomy Journal, v.53, p.279-280, 1961.

LE CLERG, E.L.; LEONARD, W.H.; CLARK, A.G. Field plot technique. Minneapolis: Burgues, 1962. 373p.

LEMOS, M.A. Variabilidade fenotípica em híbridos simples, híbridos duplos, variedade e compostos de milho (Zea mays L.). 1976, 62p. Tese (Doutorado) - Escola Superior de Agricultura Luiz de Queiroz, Piracicaba.
LESSMAN, K.J.; ATKINS, R.E. Optimum plot size and relative efficiency of lattice designs for grain sorghum yield test. Crop Science, v.3, p.477-481, 1963.

LOMA, J.L. de la. Experimentación agrícola. México: Hispano Americana, 1955. 500p.

LÚCIO, A.D. Parâmetros da precisão experimental das principais culturas anuais do Estado do Rio Grande do Sul. 1997. 64p. Dissertação (Mestrado) - Universidade Federal de Santa Maria, Santa Maria.

MARQUES, D.G. As pressuposições e a precisão dos ensaios de competição de cultivares de milho no Estado do Rio Grande do Sul. 1999. 42p. Dissertação (Mestrado) - Universidade Federal de Santa Maria, Santa Maria.

NASPOLINI FILHO, V. Variabilidade fenotípica e estabilidade em híbridos simples, híbridos duplos, variedades e compostos de milho (Zea mays L.). 1975. 68p. Dissertação (Mestrado) - Escola Superior de Agricultura Luiz de Queiroz, Piracicaba.

RESENDE, M.D.V. de; SOUZA JÚNIOR, C.L de. Número de repetições e tamanho da parcela para seleção de progênies de milho em solos sob cerrado e fértil. Pesquisa Agropecuária Brasileira, v.32, p.781-788, 1997.

SEBER, G.A.F. Linear regression analysis. NewYork: John Wiley \& Sons, 1976. 465p.

SMITH, H.F. An empirical law describing heterogeneity in the yields of agricultural crops. Journal of Agricultural Science, v.28, p.123, 1938.

STEEL, R.G.D.; TORRIE, J.H.; DICKEY, D.A. Principles and procedures of statistics. 3th. ed. Nova York: McGraw Hill, 1997. 666p.

STORCK, L. Estimativa para tamanho e forma de parcela e número de repetições para experimentos com milho (Zea mays L.). 1979. 98p. Dissertação (Mestrado) - Universidade Federal do Rio Grande do Sul, Porto Alegre.

STORCK, L.; GARCIA, D.C.; LOPES, S.J.; ESTEFANEL, V. Experimentação vegetal. Santa Maria: UFSM, 2000. 198p.

Recebido em 4 de março de 2004 e aprovado em 24 de setembro de 2004 